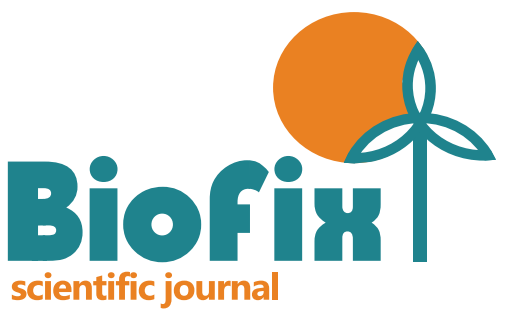

v. 2 n. 22017

Recebido em 20/09/2017

Aceito em 22/11/2017

Publicado em 29/11/2017

DOI: dx.doi.org/10.5380/biofix.v2i2.55342

\section{DINÂMICA E FRAGMENTAÇÃO FLORESTAL NA SUB-BACIA DO MÉDIO IGUAÇU (PR) NO PERÍODO DE 2000 A 2015}

\author{
DYNAMICS AND FOREST FRAGMENTATION IN THE MIDDLE IGUAÇU \\ (PR) SUB-BASIN FOR THE PERIOD 2000 TO 2015
}

\begin{abstract}
Universidade Federal do Paraná, Curitiba, Paraná, Brasil renansantos130@gmail.com ${ }^{1}$, isabela.alvarengalandim@gmail.com², anapaulacorte@gmail.com ${ }^{3}$ \& carlos_sanquetta@hotmail.com ${ }^{4}$
\end{abstract}

\section{RESUMO}

O objetivo deste trabalho foi avaliar a dinâmica e o grau de fragmentação florestal na sub-bacia do Médio Iguaçu, Paraná, no período de 2000 a 2015. Para tanto, foram utilizadas 4 imagens dos satélites Landsat 7 (2000) e 4 imagens do satélite Landsat 8 (2015), sendo estas submetidas aos procedimentos de georreferenciamento, segmentação e classificação orientada ao objeto, a fim de gerar arquivos vetoriais com as classes de interesse (Florestas Nativas e Não Florestas). A classe floresta foi classificada em 6 classes de tamanho, considerando apenas os fragmentos maiores do que 1 ha, o que foi utilizado como base para a análise de fragmentação. Analisando o período 2000-2015, foi possível identificar que as áreas de florestas naturais apresentaram uma redução, principalmente nos fragmentos maiores do que 1.000 ha localizados na área central da sub-bacia, sendo que o maior número de fragmentos está localizado na classe 1 (1-10 ha) em ambos os anos base, entretanto as maiores áreas totais são ocupadas pelas manchas com tamanho maior do que 1.000 ha. Em 2000 e 2015, a classe 6 (>1.000 ha) foi a classe mais afetada pelo efeito de borda, embora os fragmentos dessa classe retratem uma maior área protegida quando comparados a menores fragmentos. Nota-se também que a maioria dos fragmentos são alongados, apresentando formas mais irregulares conforme aumenta a classe de tamanho. Assim, pode-se concluir que a sub-bacia possui uma paisagem fragmentada, mas ainda apresenta grandes extensões de áreas que podem ser utilizadas como refúgio para a biodiversidade.

\section{ABSTRACT}

The aim of this study was to evaluate the forest dynamics and the fragmentation level in the Medium Iguaçu sub-basin, Parana, over the period 2000-2015. For this purpose, it was used four Landsat 7 and four Landsat 8 satellites images, regarding 2000 and 2015 respectively. The image scenes went through georeferencing, segmentation and object-based classification procedures in order to generate vector files with the classes of interest (Natural Forests and Non-Forest). The class Natural Forest was classified according to 6 classes of size, considering only forest patches larger than 1 ha, which also were used for the fragmentation analysis. Analyzing the period 2000-2015, it was possible to identify that natural forest areas presented a reduction in area, mainly from fragments larger than 1.000 ha located in the central region of the sub-basin. It can be noticed that the larger number of fragments are grouped in the class 1 (1-10 ha) in both base years, however, the largest total areas are occupied by classes of patches larger than 1.000 ha. In addition, in 2000 and 2015, the class 6 (>1000 ha) represented the larger total of edges, also most patches are elongated so as the class increases, more frequent irregular shapes are. Thus, it can be concluded that the subbasin has a fragmented landscape, although it is occupied by large extensions of areas, which can be used as refuge for the biodiversity.

PALAVRAS-CHAVE: Floresta, Métricas de paisagem, KEYWORDS: Forest, Landscape metrics, Remote sensing. Sensoriamento remoto. 


\section{INTRODUÇÃO}

Historicamente, os impactos da ação humana sobre o ambiente têm causado intensas alterações na paisagem florestal do Brasil, especialmente no bioma Mata Atlântica, que ainda mantém cerca de $11 \%$ de sua cobertura original (RIBEIRO et al., 2009). Mesmo com a criação de políticas para conservação e proteção dessas florestas, as queimadas e os desmatamentos são utilizados no preparo de áreas com potencial econômico (CASTELO, 2015).

Dados do projeto MAPBIOMAS (2017) mostram uma oscilação das taxas de desmatamento no estado do Paraná entre os anos de 2014 e 2016 . Diferentemente do período de 2014 a 2015, no último período avaliado (2015 a 2016) houve redução de cerca de $9 \%$ das florestas naturais do estado, que hoje ocupam uma área de aproximadamente 5.445.760 hectares. Possivelmente, isso se deve a intensa exploração das florestas de araucária, em uma situação de formação de paisagens com um alto grau de fragmentação (BROADBENT et al., 2008).

Os fragmentos florestais podem ser definidos como remanescentes isolados de floresta, formados a partir da interrupção, antrópica ou natural, de grandes e contínuas áreas de vegetação nativa (GARCIA et al., 2013). Essa fragmentação atinge diretamente o desenvolvimento de populações naturais, podendo gerar diversas consequências negativas para os ecossistemas, como a redução da biodiversidade e de variabilidade genética, por exemplo (GARCIA, 2011).

Muitos estudos sobre dinâmica e fragmentação florestal têm sido desenvolvidos por pesquisadores. Entretanto, esses estudos geralmente são abrangentes e podem não considerar as particularidades de cada região. Além disso, não há padronização dos procedimentos de análise utilizados, o que pode gerar dúvidas em relação a precisão dos valores de desmatamento a nível local (HENTZ et al., 2015a).

Ademais, há déficit de pesquisas relacionadas a área florestal da sub-bacia do Médio Iguaçu - PR, o que faz necessária a busca por dados mais precisos sobre a dinâmica e a fragmentação florestal dessa região. Diante disso, as técnicas de Ecologia da Paisagem têm sido comumente utilizadas em análises de ambientes fragmentados, uma vez que permitem uma melhor compreensão das relações espaciais e da dinâmica florestal desses habitats (BEZERRA et al., 2011).

A ecologia da paisagem é baseada em métricas ou índices de paisagem, as quais permitem descrever os padrões espaciais de forma quantitativa, bem como a própria estrutura da paisagem. As principais métricas usadas para essas análises são: área, área central, borda, densidade, forma e proximidade (BEZERRA et al., 2011; HENTZ et al., 2015a).

Normalmente, por considerarem características espaciais, as métricas de paisagem são utilizadas em conjunto com técnicas de geoprocessamento (RUDOLPHO et al., 2013), uma vez que essas possibilitam a análise de uma grande variedade de informações sobre uma determinada área, sendo, atualmente, muito utilizadas para o entendimento dos fenômenos ambientais (FONSECA et al., 2013).

Os mapas de uso e ocupação do solo geralmente são gerados a partir da interpretação visual ou pela classificação automática de imagens digitais. Para Almeida et al. (2014), apesar da aceitação das análises visuais, os procedimentos automáticos ainda têm se apresentado mais eficazes em projetos de maior proporção, pois geram resultados em maior velocidade, de forma mais simples e menos onerosa. Apesar disso, esse método geralmente apresenta problemas de confusão entre as classes de interesse. Dessa forma, a utilização simultânea dos métodos automáticos e visuais mostra-se a mais ideal (MARUJO, 2013).

As classificações tradicionalmente adotadas para as imagens de média resolução, ou seja, as classificações supervisionadas, muitas vezes, apresentam deficiências para o correto enquadramento nas classes tipológicas. Uma alternativa que tem sido adotada é a classificação orientada ao objeto pois ela leva em consideração não só a resposta espectral dos objetos, mas também características como forma, cor e textura, criando agrupamentos de pixels de acordo com a sua homogeneidade (ALMEIDA et al., 2014).

Diante desse cenário, o presente trabalho teve como propósito avaliar a dinâmica e a fragmentação florestal, por meio de métricas de fragmentos e análise de tamanho, na sub-bacia do Médio Iguaçu (PR) no período de 2000 a 2015.

\section{MATERIAL E MÉTODOS}

\section{Área de estudo}

A Bacia do Rio Iguaçu é formada por três sub-bacias: Alto Iguaçu, Médio Iguaçu e Baixo Iguaçu. Com uma área de aproximadamente $70.800 \mathrm{~km}^{2}$, a bacia do Iguaçu compreende grande parte da região Sul do estado do Paraná, além do Norte de Santa Catarina e Leste da Argentina (SEMA, 2013). A sub-bacia do Médio Iguaçu, por sua vez, representa 17.988,47 $\mathrm{km}^{2}$ de área (SUDERHSA, 
2006).

De acordo com a classificação de Köppen, a região do Médio Iguaçu apresenta clima $\mathrm{Cfb}$, caracterizado por verões amenos e temperatura média de $22^{\circ} \mathrm{C}$ nos meses mais quentes (ITCG, 2008). As formações vegetais mais comuns na região são Floresta Ombrófila Mista (FOM) Montana e Alto Montana, com presença de Campos Gerais na porção mais Oeste da sub-bacia (ITCG, 2009).

\section{Processamento das Imagens}

A análise da dinâmica e da fragmentação foi realizada a partir de oito cenas dos satélites Landsat 7 (ano de 2000) e Landsat 8 (ano de 2015), totalizando quatro cenas para cada ano base. É importante ressaltar que algumas cenas do ano 2000 apresentaram baixa qualidade, devido à elementos que dificultaram a interpretação, como a presença de nuvens.

Assim, foram selecionadas cenas de anos posteriores, buscando aproximar ao máximo da data inicial do período avaliado. As imagens apresentaram resolução de $30 \mathrm{~m}$ e a composição digital adotada foi RGB:233. As órbitas-ponto utilizadas no trabalho foram: 222/077 (04/12/2003 e $18 / 01 / 2016), 222 / 078$ (12/04/2003 e 01/08/2016), $221 / 078 \quad(12 / 08 / 2000$ e $02 / 08 / 2016), \quad 221 / 079$ (17/03/2002 e 16/04/2016). Os dados foram obtidos no portal eletrônico do Instituto Nacional de Pesquisas Espaciais (INPE).

Para a correção geométrica das cenas, o procedimento foi realizado adotando as imagens de 2006 e 2007 do satélite SPOT 5 como base, as quais foram ortorretificadas e disponibilizadas pelo Instituto de Terras, Cartografia e Geologia do Paraná (ITCG). O georreferenciamento foi realizado no software $\operatorname{ArcGis}^{\circledR}$ versão 10.4, sendo coletados aproximadamente 20 pontos de controle por cena.

Para o georreferenciamento, o software ArcGIS utiliza um polinômio baseado em pontos de controle e um algoritmo de Ajuste de Mínimos Quadrados (LSF, em inglês), conforme ESRI (2016). Em seguida, as imagens foram recortadas para o limite de abrangência da área de interesse e adicionadas bordaduras de $20 \mathrm{~km}$ como margem de segurança.

\section{Classificação orientada a objeto}

As imagens georreferenciadas foram segmentadas com o uso do software Definiens Professional 5.01, o que permitiu o agrupamento dos pixels em segmentos em um primeiro processamento. Para a segmentação, foram utilizados os parâmetros fator de escala (20) e peso para cor, forma, compacidade e textura $(0,9 ; 0,1 ; 0,5$ e 0,5 , respectivamente).

Posteriormente, procedeu-se a classificação orientada a objeto. Para isso, o primeiro nível da hierarquia foi estruturado considerando as classes: 1 ) áreas de floresta $\mathrm{e}$ 2) não-floresta. Posteriormente, criou-se um novo nível, considerando a classe 1) floresta do primeiro nível, dividida em dois níveis, sendo: 1.a) florestas nativas (estágios inicial, médio e avançado) 1.b) áreas de reflorestamento.

Foram estabelecidos critérios de classificação de acordo com o comportamento espectral dos alvos imageados na composição RGB determinada (233). Para a classe de florestas nativas, foram consideradas como áreas de vegetação secundária em estágio inicial aquelas que apresentaram textura fina, coloração vermelho claro e sem forma definida, enquanto áreas de vegetação secundária em estágio médio e avançado apresentaram textura rugosa, coloração vermelho-médio à vermelho escuro e sem forma definida.

As áreas de textura lisa, coloração vermelho médio à preto e forma reta foram classificadas como áreas de reflorestamento. Os alvos que apresentaram comportamento espectral diferenciado dos alvos das classes estabelecidas foram classificados como áreas de não floresta, como, por exemplo, áreas de agricultura, água, pecuária e áreas urbanas.

Por fim, foram gerados arquivos vetoriais (shapefiles), os quais foram submetidos a checagem e reambulação no software $\operatorname{ArcGIS}^{\circledR} 10.4$, em função de possíveis erros que durante a classificação. Nessa fase, os shapefiles foram também confrontados com o perímetro da área de interesse, o que possibilitou analisar apenas a área útil da sub-bacia.

\section{Análise do grau de fragmentação}

Para as análises do grau de fragmentação, foi considerada apenas a classe de florestas nativas. Os polígonos foram agrupados em classes de tamanho, considerando apenas aqueles com maiores que um hectare. Essa decisão foi baseada no conceito de florestas da United Nations Framework Convention on Climate Change (UNFCCC, 2001).

A definição das classes foi realizada adotando as propostas de Ribeiro et al. (2009) e Hentz et al. (2015b), sendo apresentadas da seguinte forma: classe $1: 1 \mathrm{H} 10$ ha; classe 2: $10 \dashv 40$ ha; classe $3: 40 \dashv 100$ ha; classe 4: 100 $\dashv 500$ ha; classe 5: $500 \dashv 1.000$ ha; e classe 6: $>1.000$ ha.

A partir das classes de tamanho, foi possível analisar a 
distribuição dos fragmentos na sub-bacia para os anos de 2000 e 2015, os quais também possibilitaram a geração de um mapa com as mudanças ocorridas no período avaliado. O mapa de ganho e perdas foi criado a partir da união dos shapefiles de fragmentos dos dois anos base.

A partir disso, utilizando a tabela de atributo do shapefile gerado, identificou-se os polígonos que apresentaram mudança, sendo aqueles classificados anteriormente como floresta em 2000 e, posteriormente, como não-floresta em 2015 (perda), e vice-versa (ganho). Após o cálculo de áreas, foi aplicado o teste de quiquadrado $\left(\chi^{2}\right)$, considerando a probabilidade de $95 \%$ e cinco graus de liberdade.

Os cálculos das métricas foram realizados com a extensão Patch Analyst, considerando características como área e densidade, borda e forma. A descrição das métricas foi adaptada de Hentz (2015) e apresentada na Tabela 1.

Tabela 1. Métricas de paisagem utilizadas para a análise de fragmentação na sub-bacia do Médio Iguaçu

\begin{tabular}{|c|c|c|c|c|c|}
\hline Grupo & Métrica & Sigla & Unidade & Descrição & Fórmula \\
\hline \multirow{6}{*}{$\begin{array}{l}\frac{0}{0} \\
\frac{\pi}{0} \\
\frac{0}{n} \\
\frac{1}{0} \\
0 \\
0 \\
0 \\
\mathbb{0} \\
\frac{1}{4}\end{array}$} & Área da classe & CA & ha & $\begin{array}{l}\text { Soma das áreas de todos os } \\
\text { fragmentos da classe }\end{array}$ & $C A=\sum_{i=1}^{n} C_{i}$ \\
\hline & Tamanho médio & MPS & ha & $\begin{array}{l}\text { Razão entre a soma das áreas dos } \\
\text { fragmentos e o número de } \\
\text { fragmentos }\end{array}$ & $M P S=\frac{\sum_{j=1}^{n} a_{i j}}{n_{i}}$ \\
\hline & $\begin{array}{l}\text { Mediana da } \\
\text { classe }\end{array}$ & MedPS & ha & $\begin{array}{l}\text { O tamanho do fragmento que se } \\
\text { encontra no meio do conjunto de } \\
\text { dados, ou seja, na mediana }\end{array}$ & - \\
\hline & $\begin{array}{l}\text { Número de } \\
\text { Fragmentos }\end{array}$ & NUMP & - & $\begin{array}{l}\text { Número total de fragmentos } \\
\text { encontrados em uma classe }\end{array}$ & $N U M P=\sum n_{i}$ \\
\hline & $\begin{array}{l}\text { Desvio padrão } \\
\text { do tamanho }\end{array}$ & PSSD & ha & $\begin{array}{l}\text { Dispersão dos tamanhos dos } \\
\text { fragmentos em relação à média }\end{array}$ & $P S S D=\frac{\sum_{j=1}^{n}\left|a_{i j}-\left(\frac{\sum_{j=1}^{n} a_{i j}}{n_{i}}\right)\right|^{2}}{n}$ \\
\hline & $\begin{array}{l}\text { Coeficiente de } \\
\text { variação de área }\end{array}$ & PScov & $\%$ & $\begin{array}{c}\text { Razão entre o desvio padrão do } \\
\text { tamanho e o tamanho médio, } \\
\text { multiplicado por } 100\end{array}$ & $P S \operatorname{cov}=\frac{P S S D}{M P S}$ \\
\hline \multirow{2}{*}{ 웅 } & Total de bordas & TE & $\mathrm{m}$ & $\begin{array}{c}\text { Soma dos perímetros dos } \\
\text { fragmentos }\end{array}$ & $T E=\sum_{i=1}^{n} e_{i}$ \\
\hline & $\begin{array}{l}\text { Densidade de } \\
\text { bordas }\end{array}$ & ED & $\mathrm{m} \mathrm{ha}{ }^{-1}$ & $\begin{array}{c}\text { Total de bordas da classe em relação } \\
\text { a paisagem }\end{array}$ & $E D=\frac{T E}{C A}$ \\
\hline \multirow{3}{*}{ ํㅗㄴ } & $\begin{array}{l}\text { Índice de forma } \\
\text { médio }\end{array}$ & MSI & - & $\begin{array}{l}\text { Soma do perímetro dos fragmentos } \\
\text { dividido pela raiz quadrada da área, } \\
\text { ajustada para padrão circular, } \\
\text { ponderado por no de fragmentos da } \\
\text { classe }\end{array}$ & $P S S D=\frac{\frac{\sum_{j=1}^{n}\left(0,25 p_{i j}\right)}{\int a_{i j}}}{n_{i}}$ \\
\hline & $\begin{array}{l}\text { Dimensão fractal } \\
\text { da mancha } \\
\text { média }\end{array}$ & MPFD & - & $\begin{array}{c}\text { Multiplicação de } 2 \text { vezes o logaritmo } \\
\text { do perímetro, dividido pelo } \\
\text { logaritmo da área do fragmento }\end{array}$ & $M P F D=\frac{2 x \log e_{i}}{\log C_{i}}$ \\
\hline & $\begin{array}{l}\text { Razão } \\
\text { perímetro-área }\end{array}$ & MPAR & $m h^{-1}$ & $\begin{array}{l}\text { Soma de cada relação perímetro- } \\
\text { área dividido pelo número de } \\
\text { fragmentos }\end{array}$ & $M P A R=\frac{\sum_{I=1}^{n}\left(\frac{e_{i}}{C i}\right)}{n}$ \\
\hline
\end{tabular}

Fonte: Adaptado de Hentz (2015).

\section{RESULTADOS E DISCUSSÃO}

Para o cálculo da área total de florestas nativas, foram considerados todos os tamanhos de fragmentos. De forma geral, houve mudanças negativas nas áreas de floresta nativa na sub-bacia do Médio Iguaçu. Apesar da predominância de florestas secundárias na região, essa classe sofreu redução de área de cerca de $13,6 \%$, variando de 1.120.974,75 ha em 2000 para 968.649,67 ha em 2015.
$\mathrm{Na}$ Tabela 2, pode-se observar os valores de área desconsiderando os fragmentos menores que um hectare. No ano de 2015, a área de fragmentos foi menor quando comparada ao ano de 2000, sendo condição aos fragmentos menores do que um hectare. Percebe-se também que, à medida que a classe de tamanho aumentou, houve acréscimo da área total ocupada, em que a classe 6 representou de 840 a 995 mil hectares e a classe 1 variou entre 14 e 20 mil ha. 
Tabela 2. Áreas e porcentagens de fragmentos na sub-bacia do Médio Iguaçu no período de 2000-2015

\begin{tabular}{ccccccccc}
\hline \multirow{2}{*}{ Classe } & \multirow{2}{*}{ Amplitude } & \multicolumn{2}{c}{ Ano de 2000 } & \multicolumn{2}{c}{ Ano de 2015 } & \multicolumn{2}{c}{ Mudança (2015-2000) } & \multirow{2}{*}{$\boldsymbol{p}$} \\
\cline { 3 - 7 } & & Área (ha) & \% & Área (ha) & \% & Área (ha) & $\%$ & \\
\hline 1 & 1 H 10 ha & $14.137,38$ & $1,26 \%$ & $20.621,01$ & $2,13 \%$ & $6.483,63$ & $45,86 \%$ & 2973,50 \\
\hline 2 & $10 \dashv 40$ ha & $21.245,75$ & $1,90 \%$ & $24.872,81$ & $2,57 \%$ & $3.626,96$ & $17,07 \%$ & 619,21 \\
3 & $40 \dashv 100$ ha & $20.362,96$ & $1,82 \%$ & $21.207,35$ & $2,19 \%$ & 844,39 & $4,15 \%$ & 35,01 \\
\hline 4 & $100 \dashv 500$ ha & $43.627,84$ & $3,89 \%$ & $39.190,90$ & $4,05 \%$ & $-4.436,94$ & $-10,17 \%$ & 451,24 \\
\hline 5 & $500 \dashv 1.000$ ha & $26.355,46$ & $2,35 \%$ & $20.361,20$ & $2,11 \%$ & $-5.994,26$ & $-22,74 \%$ & 1363,33 \\
\hline 6 & $>1.000$ ha & $994.582,53$ & $88,78 \%$ & $840.306,82$ & $86,94 \%$ & $-154.272,91$ & $-15,51 \%$ & 23930,64 \\
\hline & Total & $1.120 .311,91$ & $100 \%$ & $996.559,98$ & $100 \%$ & & & 29372,92 \\
\hline
\end{tabular}

Valor de $p$ equivale ao somatório da razão entre a diferença das áreas ao quadrado e a área do ano 2000.

Somatório de $p$ representa valor $\chi^{2}$. O valor de $\chi^{2}$ tabelado, considerando $95 \%$ de probabilidade e cinco graus de liberdade, foi de 1,145.

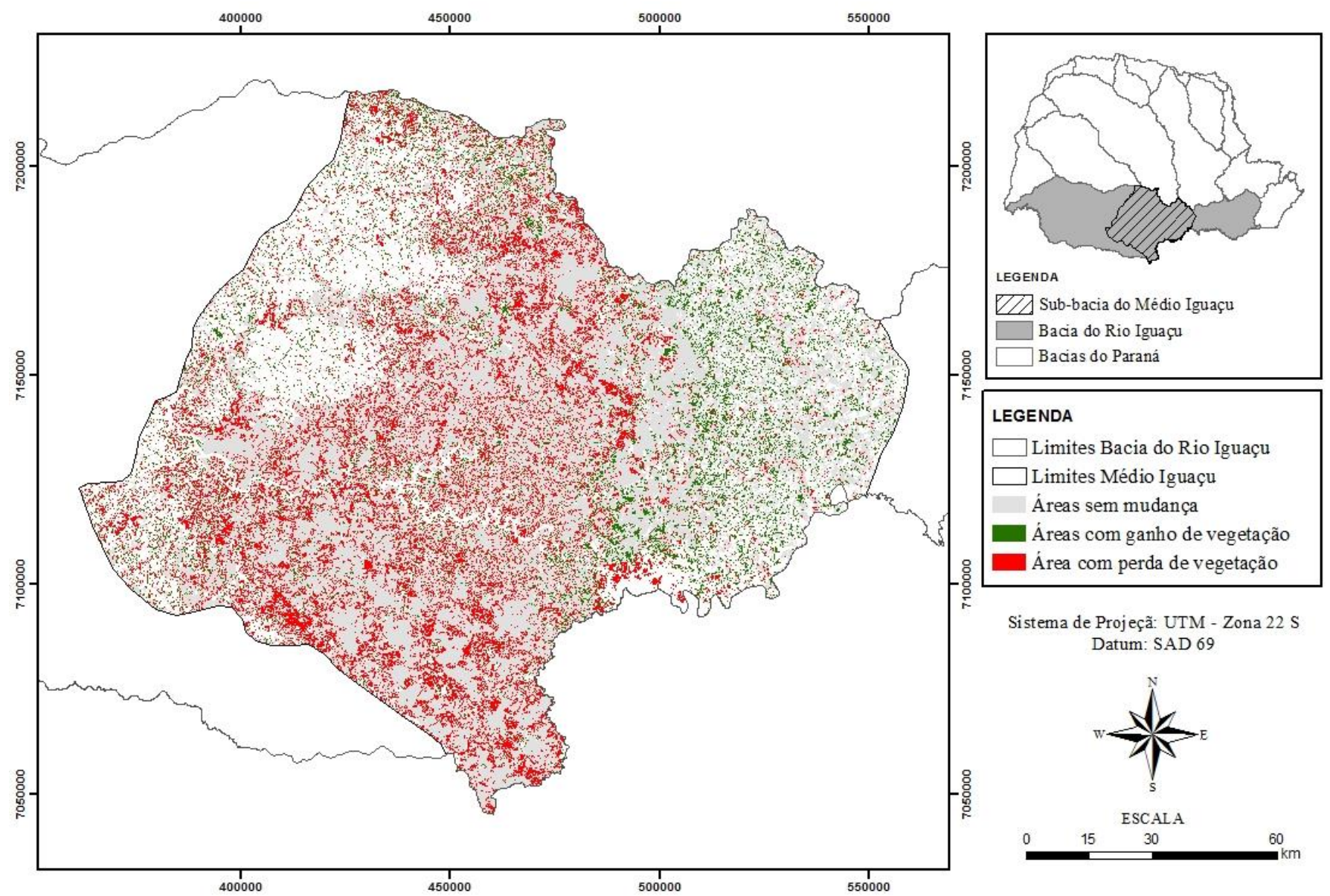

Figura 1. Áreas de ganho e perda de florestas nativas na sub-bacia do Médio Iguaçu no período de 2000-2015.

Nota-se que as menores classes tiveram acréscimo de área em 2015, com destaque a classe de 1 a 10 ha, que obteve aumento de $6.483,63$ ha, sendo equivalente a $45,86 \%$. Contrariamente, as classes 4,5 e 6 apresentaram valores menores de área no segundo ano base, sendo que a classe 5 obteve redução de aproximadamente 5.944 ha, apontando a maior perda em porcentagem entre as classes de maior tamanho.

Apesar disso, a classe 6, por ocupar grande extensão territorial, apresentou significativas perdas, de aproximadamente de 154 mil há, entre os anos 2000 e 2015. Ademais, no ano de 2000 , a maior proporção de área se concentrou nas três maiores classes de tamanho, enquanto em 2015 as classes 2, 4 e 6 possuíam as maiores porcentagens, representando cerca de $94 \%$ da área total de fragmentos.

Pode-se observar que o valor de $\chi^{2}$ foi de 29.372,92, sendo menor ao tabelado de 1,145, indicando diferença 
significativa entre as áreas de floresta dos dois anos base. Para uma melhor visualização das diferenças entre os anos, um mapa com a distribuição das perdas e ganhos de vegetação entre 2000 e 2015 é apresentado na Figura 1.

As perdas de florestas foram mais intensas na região
Central e Oeste da sub-bacia, enquanto os ganhos se concentraram na região Leste, reforçando a evidência de que a região Oeste da sub-bacia foi a que mais sofreu com os processos de fragmentação.
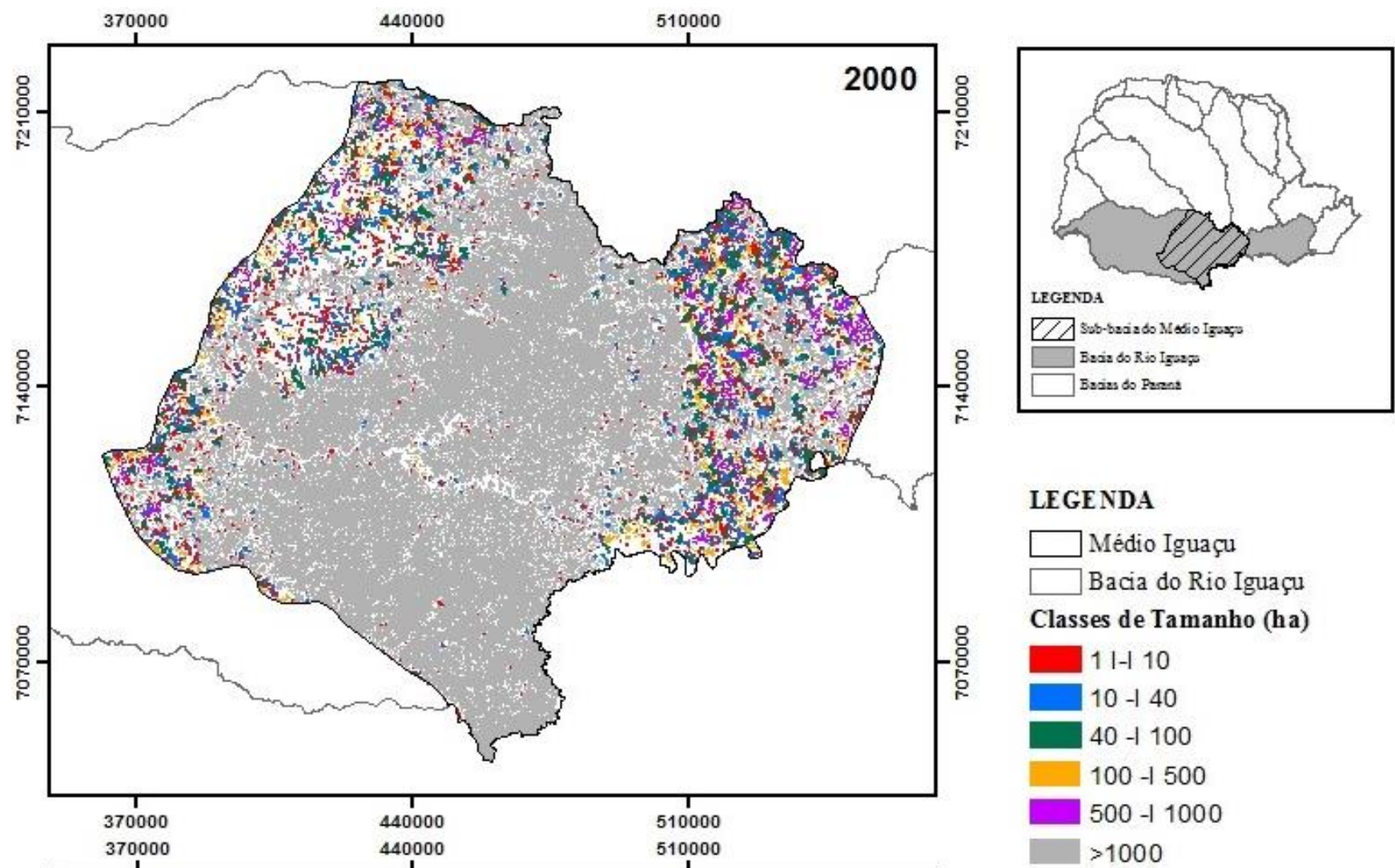

\section{LEGENDA}

$\square$ Médio Iguaçu Bacia do Rio Iguaçu

Classes de Tamanho (ha)

- 1 I-I 10

$10-10$

$40-100$

$100-1500$

$500-1000$

$>1000$
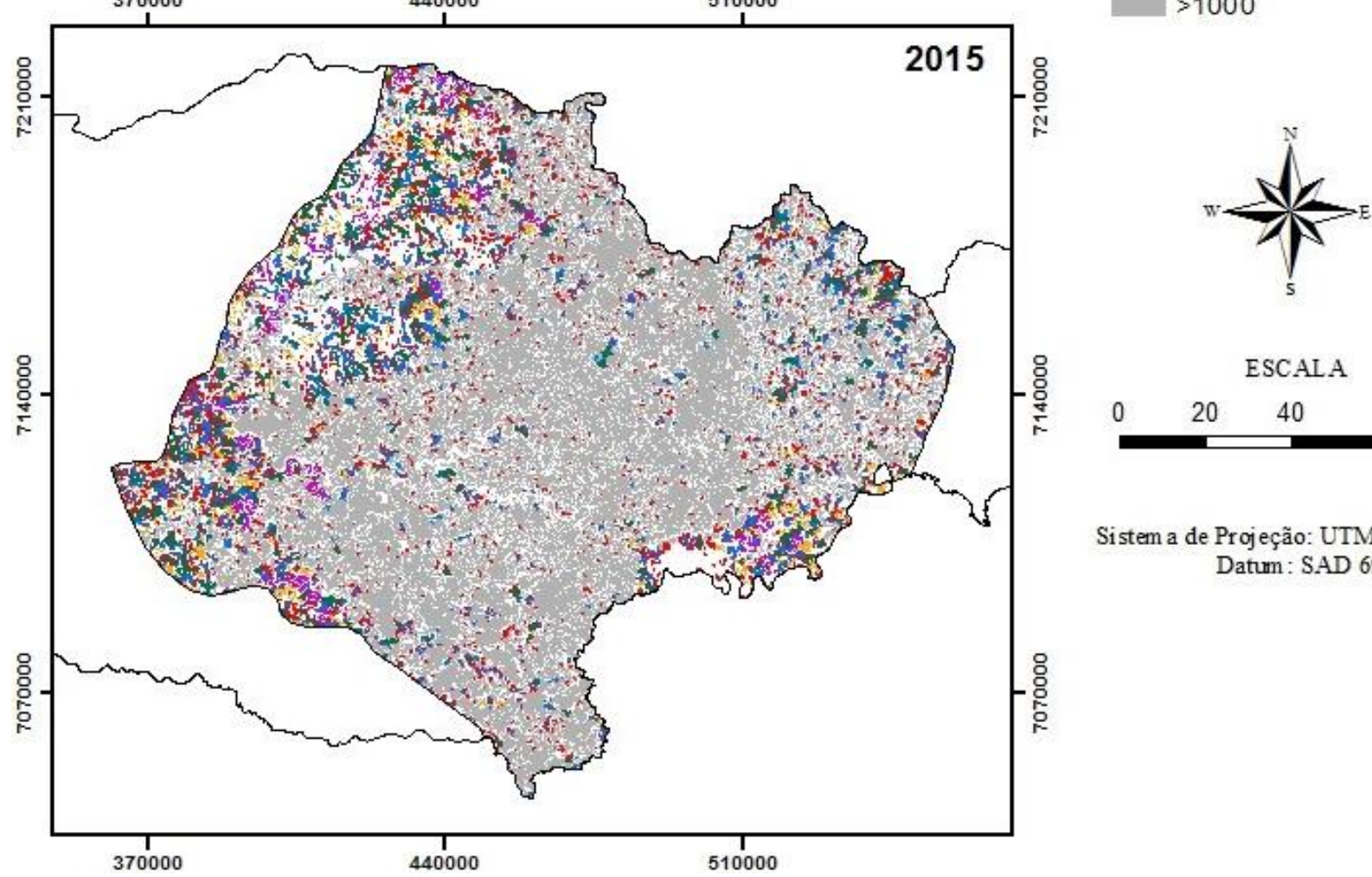

ESCALA

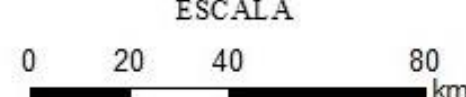

Sistem a de Projeção: UTM - Zona 22 S Datum: SAD 69

Figura 2. Distribuição das classes de tamanho dos fragmentos na sub-bacia do médio Iguaçu no período de 2000-2015. 
Ademais, comparando-se as Figuras 1 e 2, percebe-se também que, devido a sua localização, a classe de tamanho de fragmentos maiores do que 1.000 ha foi a que mais sofreu redução em área entre os anos 2000 e 2015. Da mesma forma, a região Leste da sub-bacia foi a que mais registrou ganhos de floresta nos 15 anos avaliados. Essas mudanças também influenciaram na distribuição das classes de tamanho de fragmentos, a qual pode ser visualizada na Figura 2.

Observa-se na Figura 2 que, em ambos os anos, a classe de fragmentos com tamanho maior do que 1.000 ha era predominante, sendo que sua distribuição se dava principalmente na área central da sub-bacia. Por outro lado, nota-se mudanças em relação a distribuição das demais classes. Em 2000, havia uma grande concentração de fragmentos com área entre 1 e 1.000 ha, principalmente, nas regiões leste e noroeste da sub-bacia. Já no ano de 2015, pode-se notar que a região leste estava ocupada, em sua maioria, por fragmentos maiores do 1.000 ha (ganho de floresta), enquanto a região oeste apresentava-se mais fragmentada, com fragmentos menores do que 1.000 ha (perda de floresta).

Em relação a análise da paisagem, os cálculos das métricas para cada classe de tamanho estão dispostos nas Tabelas 3 e 4. Na Tabela 3, estão apresentadas as métricas relacionadas a área e densidade e borda. Novamente, pode-se verificar crescimento da área total (CA) de acordo com o aumento da classe de tamanho em ambos os anos, com exceção da classe 5 . A mesma tendência pode ser identificada nos valores de tamanho médio e mediana das classes (MPS e MedPS).

Por outro lado, o número de fragmentos (NumP) diminuiu conforme a classe variou da menor para a maior. No ano de $2000,70 \%$ dos fragmentos estavam na classe 1 , enquanto apenas $0,5 \%$ das manchas tinham tamanho maior do que 1000 ha. Da mesma forma, em 2015, 75\% dos fragmentos estavam agrupados na menor classe e apenas $0,4 \%$ na classe 6 .

No ano de 2000, o menor fragmento da classe 6 possuía área de 1.015,1 ha, enquanto o tamanho do maior fragmento foi de $531.085,8$ ha. No último ano base, os tamanhos de fragmentos da classe 6 variaram entre $1.037,3$ e 489.835,6, o que demonstra elevada amplitude entre os maiores e menores fragmentos. Em relação as mudanças no período de 2000 a 2015, destaca-se o aumento considerável do número de manchas na classe 1 , uma vez que essa classe apresentou diferença de 2.040 fragmentos, sendo de aproximadamente $50 \%$.

Tanto no ano de 2000, quanto em 2015, a classe 6 demonstrou o total de bordas mais expressivo em função do maior tamanho de áreas, sendo de $36.020 .825,5 \mathrm{~m}$ e 64.069.657,10 m, respectivamente. Porém, ao verificar o desenvolvimento da classe durante o período, percebe-se mudança significativa em relação a soma dos perímetros dos fragmentos, uma vez que o ano final de avaliação teve um de bordas duas vezes maior do que em 2000.

O contrário ocorreu com a classe 5 , a qual, mesmo sendo ocupada por grandes fragmentos (entre 500 e 1.000 ha), apresentou o menor total de bordas nos dois anos base, entre 2,5 e 2,9 milhões de metros. A densidade de bordas (ED), em ambos os anos, seguiu a mesma tendência, sendo que o maior valor está relacionado a classe de tamanho $6\left(66,3 \mathrm{~m} \mathrm{ha}^{-1}\right)$ e o menor a classe $5(2,6$ $\mathrm{m} \mathrm{ha}^{-1}$ ).

Tabela 3. Métricas de paisagem de área e densidade para a sub-bacia do Médio Iguaçu no período de 2000-2015

\begin{tabular}{|c|c|c|c|c|c|c|c|c|c|}
\hline \multirow{2}{*}{ Classe } & \multirow{2}{*}{ Amplitude } & \multicolumn{6}{|c|}{ Área e densidade } & \multicolumn{2}{|l|}{ Borda } \\
\hline & & CA & MPS & MedPS & NumP & PSSD & PSCov & TE & ED \\
\hline \multicolumn{10}{|c|}{ Ano de 2000} \\
\hline 1 & $1 \mathrm{H} 10$ ha & $14.137,38$ & 3,7 & 3,00 & 3.853 & 2,28 & 62,06 & $4.600 .071,8$ & 4,1 \\
\hline 2 & $10 \dashv 40$ ha & $21.245,75$ & 19,4 & 16,70 & 1.098 & 8,15 & 42,14 & $4.278 .776,0$ & 3,8 \\
\hline 3 & $40 \dashv 100$ ha & $20.362,96$ & 64,0 & 62,20 & 318 & 16,95 & 26,46 & $3.214 .928,5$ & 2,9 \\
\hline 4 & $100 \dashv 500$ ha & $43.627,84$ & 201,1 & 165,00 & 217 & 97,18 & 48,34 & $5.615 .654,4$ & 5,0 \\
\hline 5 & $500 \dashv 1.000$ ha & $26.355,46$ & 675,8 & 681,60 & 39 & 119,42 & 17,67 & $2.923 .970,8$ & 2,6 \\
\hline 6 & $>1.000$ ha & $994.582,53$ & $36.836,4$ & $2.512,10$ & 27 & $123.156,38$ & 334,33 & $36.020 .825,5$ & 32,1 \\
\hline \multicolumn{10}{|c|}{ Ano de 2015} \\
\hline 1 & $1 \mathrm{H} 10$ ha & $20.621,01$ & 2,3 & 2,7 & 5.893 & 2,25 & 64,38 & $6.952 .686,2$ & 7,1 \\
\hline 2 & $10 \dashv 40$ ha & $24.872,71$ & 19,4 & 16,7 & 1.283 & 8,02 & 41,38 & $5.457 .727,3$ & 5,7 \\
\hline 3 & $40 \dashv 100$ ha & $21.207,35$ & 62,7 & 59,8 & 338 & 16,36 & 26,08 & $3.568 .499,9$ & 3,7 \\
\hline 4 & $100 \dashv 500$ ha & $39.190,90$ & $2.006,3$ & 182,8 & 190 & 94,90 & 46,01 & $5.585 .800,5$ & 5,8 \\
\hline 5 & $500 \dashv 1.000$ ha & $20.361,20$ & 702,1 & 681,1 & 29 & 147,12 & 20,95 & $2.545 .738,5$ & 2,6 \\
\hline 6 & $>1.000$ ha & $840.306,82$ & $30.011,0$ & $1.786,8$ & 28 & $101.637,34$ & 338,67 & $64.069 .657,10$ & 66,3 \\
\hline
\end{tabular}

$\mathrm{CA}=$ área da classe (ha); MPS = tamanho médio (ha); MedPS = mediana da classe (ha); NumP = número de fragmentos; PSSD = desvio padrão do tamanho (ha); PSCov = coeficiente de variação área (\%); TE = total de bordas (m); e ED = densidade de bordas $\left(m \mathrm{~m}^{-1}\right)$. 
Tabela 3. Métricas de paisagem de forma para a sub-bacia do Médio Iguaçu no período de 2000-2015

\begin{tabular}{cccccccc}
\hline \multirow{2}{*}{ Classe } & \multirow{3}{*}{ Amplitude } & \multicolumn{3}{c}{ Ano de 2000 } & \multicolumn{3}{c}{ Ano de 2015 } \\
\cline { 3 - 8 } & & \multicolumn{3}{c}{ Morma } \\
\cline { 3 - 8 } & $1+10$ ha & 1,8 & 1,35 & 370,1 & 1,8 & 1,36 & 379,1 \\
\hline 1 & $10 \dashv 40$ ha & 2,5 & 1,36 & 210,8 & 2,7 & 1,37 & 227,7 \\
2 & $40 \dashv 100$ ha & 3,6 & 1,37 & 160,6 & 3,8 & 1,38 & 169,7 \\
3 & $100 \dashv 500$ ha & 5,1 & 1,39 & 133,5 & 5,8 & 1,41 & 148,4 \\
4 & $500-1.000$ ha & 8,2 & 1,43 & 112,2 & 9,3 & 1,44 & 126,3 \\
5 & $>1.000$ ha & 17,3 & 1,45 & 91,9 & 21,8 & 1,46 & 97,6 \\
\hline
\end{tabular}

$\mathrm{MSI}$ = índice de forma médio; MPFD = dimensão fractal da mancha média; e MPAR = razão perímetro-área $\left(\mathrm{m} \mathrm{ha}{ }^{-1}\right)$.

A forma também é um fator essencial para a análise de fragmentação. O cálculo do índice de forma médio (MSI) possibilita comparar os fragmentos a uma forma circular. Dessa forma, quanto mais próximo de 1 for o MSI, mais circulares (ou regulares) são os fragmentos. Na Tabela 4, é possível notar que o índice de forma médio foi alto para todas as classes nos dois anos avaliados. Assim, tantos as menores quanto as maiores manchas apresentaram características irregulares.

Além disso, pode-se observar que os fragmentos das menores classes são mais circulares do que aqueles com maiores dimensões pois, apesar de alto, o MSI para aquelas classes é mais próximo a 1 . Isso é evidente ao analisar-se a classe 6, a qual apresentou valores de forma, em termos médios, próximos a 20 nos dois anos de análise.

Da mesma forma que o MSI, a dimensão fractal (MPFD) também diz respeito ao formato do fragmento, cujo valor varia de 1 a 2 , sendo que valores maiores são menos regulares. Nesse caso, a dimensão fractal aumentou de acordo com o aumento das classes em 2000 e 2015, sendo que os menores e maiores valores encontrados foram de 1,35 e 1,36 e 1,45 e 1,46, respectivamente.

A razão perímetro-área (MPAR) demonstrou pequena diferença entre os anos base, mantendo a mesma tendência nos dois anos. Diferentemente de outras métricas de forma, a MPAR se apresenta decrescente em relação as classes de tamanho. Na Tabela 4, pode-se ver que, quanto maior é a classe de tamanho, menor é a razão perímetro-área.

A redução de áreas de florestas naturais do Médio Iguaçu entre 2000 e 2015 está relacionada a diminuição em áreas dos fragmentos maiores do que 1.000 ha, que deram origem as paisagens mais fragmentadas no ano de 2015, como na região Oeste da sub-bacia, que apresentou maior presença de classes de menor tamanho. Por outro lado, o aumento do tamanho dos fragmentos na região Leste da sub-bacia pode ser explicado pela influência das leis ambientais sobre a manutenção das propriedades rurais (CALEGARI et al., 2010).
A diminuição em área de fragmentos florestais pode causar uma série de consequências, como a redução da biodiversidade local. $\mathrm{O}$ decrescimento das áreas de fragmentos causa a locomoção da biodiversidade, que, devido à baixa oferta de recursos, se desloca para outros locais, causando, em alguns casos, excedentes populacionais.

Esses excedentes, por sua vez, causam competição entre as populações, gerando desequilíbrio nas taxas de sobrevivência e reprodução. Em outras situações, as populações podem ainda se locomover para manchas de menor qualidade, como para locais desfavoráveis ao desenvolvimento (RAMBALDI et al., 2003).

Para Silva e Souza (2014), as métricas de tamanho e densidade de fragmentos, apesar de serem atributos simples de se calcular, são indispensáveis para a estimativa de outras características da paisagem, como as de forma e de borda. Neste estudo, foi identificado que maiores áreas totais de fragmento ocupam maiores classes de tamanho, demonstrando relação positiva entre a área dos fragmentos e as classes de tamanho.

Da mesma forma, o número de fragmentos foi inversamente proporcional a classe de tamanho, ou seja, enquanto as maiores classes apresentaram menor número de fragmentos, as menores possuíam maiores quantidades de manchas. Essa tendência também foi observada por Pirovani et al. (2014) em uma bacia hidrográfica do estado do Espírito Santo.

Além disso, foi identificado aumento de aproximadamente $50 \%$ no número de fragmentos da classe 1 . Isso pode estar relacionado as perdas em áreas de classes de maior tamanho, como na classe 6 (> 1.000 ha). Calegari et al. (2010) citam que pequenos fragmentos florestais podem ser utilizados como pontos de ligação ecológico que auxiliam a locomoção da fauna entre os fragmentos.

Em relação ao desvio padrão e ao coeficiente de variação (PSSD e PScov), a classe 6 (> 100 ha) apresentou valores altos para ambos os anos base. Isso aconteceu 
devido a grande diferença de tamanho entre os fragmentos da classe (JUVANHOL et al., 2011). Ao estudar o desvio padrão da área dos fragmentos maiores do que 1.000 ha na Bacia do Rio Iguaçu (PR), Hentz et al. (2015a) também identificaram valores altos para os dois atributos, com coeficiente de variação de $318,90 \%$ e próximo ao deste trabalho.

Os maiores valores para total e densidade de bordas (TE e ED) estão relacionados a classe de maior tamanho (classe 6), destacando-se a mudança ocorrida nessa classe entre os anos de 2000 e 2015. Devido a sua área e ao seu formato mais irregular, os fragmentos com tamanho maior do que 1.000 ha são também aqueles que mais sofrem com o efeito de borda.

Porém, considerando a área núcleo, esses fragmentos apresentam maior área protegida em relação aos fragmentos de menor tamanho (PIROVANI et al., 2014). Segundo Ribeiro et al. (2009), pelo menos 50\% da Mata Atlântica está localizada a menos de $100 \mathrm{~m}$ de áreas abertas, sendo propícias a influências do efeito de borda. Para essas situações, Hentz et al. (2015a) sugerem que sejam desenvolvidas ações de conservação, com o intuito de diminuir a interação entre os fragmentos florestais e os fatores externos, como pragas e incêndios.

As análises dos índices de forma médio (MSI) e dimensão fractal (MPFD) apontaram que, quanto maior a classe de tamanho, mais irregulares são os fragmentos, demonstrando também pouca mudança nos 15 anos avaliados. Calegari et al. (2010) também encontrou pequenas mudanças de forma no período avaliado.

Apesar disso, em seu estudo, Calegari et al. (2010) não notaram grande irregularidade nos fragmentos, o que os caracteriza com uma forma simples. Hentz et al. (2015a), por sua vez, verificaram que classes de tamanho maior apresentaram forma mais irregular, sendo que todas os tamanhos mostraram MSI alto.

Os valores de perímetro/áreas médios (MPAR) não demonstraram grande diferença entre os anos base e apresentaram a mesma tendência em relação as classes de tamanho, em que a razão perímetro-área foi inferior nas maiores classes. Esse comportamento também foi verificado por Castro (2008), que identificou que as áreas de borda tendem a influenciar mais facilmente fragmentos menores e mais irregulares.

\section{CONCLUSÕES}

As áreas de florestas nativas demonstram redução, principalmente nas classes de fragmentos superiores a 1.000 ha. Entretanto, não são observadas grandes diferenças nas métricas de área e densidade, borda e forma durante o período avaliado, com exceção da área da classe e soma de perímetros das classes 1 e 6, respectivamente, as quais se mostram maiores no ano de 2015.

Atualmente, as áreas mais fragmentadas estão localizadas na região Oeste da sub-bacia do Médio Iguaçu, as quais, apesar de sofrerem maior influência do efeito de borda, podem ser utilizadas como pontes ecológicas entre grandes fragmentos. Ainda, pode-se notar a presença de fragmentos da classe 6 com maior ocorrência na região central as sub-bacia, o que provavelmente mantêm a maior parte da biodiversidade local.

Há grande déficit de informações sobre a dinâmica e a fragmentação florestal na região do Médio Iguaçu. Dessa forma, sugere-se posteriores estudos que definam as áreas de estágio inicial e médio-avançado de vegetação, bem como o cálculo de outras métricas de paisagem, como área central e proximidade, a fim de identificar as reais áreas de conservação da fauna e da flora e compreender as suas interações.

Apesar de demonstrar melhor desempenho, quando comparada aos outros tipos de classificação, a classificação orientada a objeto ainda apresenta confusões ao enquadrar as tipologias. Assim, pode-se examinar valores menores de fator de escala na etapa de segmentação, visando obter objetos menores e, consequentemente, melhor resolução da classificação.

\section{REFERÊNCIAS}

ALMEIDA, A. S.; WERNECK, G. L.; RESENDES, A. P. C. Classificação orientada a objeto de imagens de sensoriamento remoto em estudos epidemiológicos sobre leishmaniose visceral em área urbana. Cadernos de Saúde Pública, v. 30, n. 8, p. 1639-1653, 2014.

BEZERRA, C. G.; SANTOS, A. R.; PIROVANI, D. B.; PIMENTEL, L. B.; EUGENIO, F. C. Estudo da fragmentação florestal e ecologia da paisagem na sub-bacia hidrográfica do Córrego Horizonte, Alegre, ES. Espaço \& Geografia, v. 14, n. 2, p. 257-277, 2011.

BROADBENT, E. N.; ASNER, G. P.; KELLER, M.; KNAPP, D. E.; OLIVEIRA, P. J. C.; SILVA, J. N. Forest fragmentation and edge effects from deforestation and selective logging in the Brazilian Amazon. Biological Conservation, v. 141, p. 1745-1757, 2008.

CALEGARI, L.; MARTINS, S. V.; GLERIANI, J. M.; SILVA, E.; BUSATO, L. C. Análise da dinâmica de fragmentos florestais no município de Carandaí, MG, para fins de restauração florestal. Revista Árvore, v. 34, n. 5, p. 871-880, 2010.

CASTELO, T. B. Legislação florestal brasileira e políticas do governo de combate ao desmatamento da Amazônia Legal. Ambiente \& Sociedade, v. 18, n. 4, p. 221-242, 2015. 
CASTRO, D. M. Efeito de borda em ecossistemas tropicais: síntese bibliográfica e estudo de caso em fragmentos de Cerrado, na região Nordeste do Estado de São Paulo. 2008. 171 f. Dissertação (Mestrado em Ciências de Ecossistemas) Universidade de São Paulo, São Paulo, 2008.

ESRI - Environmental Systems Research Institute. ArcGIS ${ }^{\circledR} \mathbf{1 0 . 4}$ Help. 2016. Disponível em: <http://desktop.arcgis.com/en/ arcmap/10.4/get-started/main/get-started-with-arcmap.htm>. Acesso em: 20/05/2017.

FONSECA, S. M.; SANTOS, D. C.; HERMANO, V. M. Geoprocessamento aplicado à análise dos impactos socioambientais urbanos: estudo de caso do bairro Santo Expedito em Buritizeiro/MG. Revista de Geografia, v. 30, n. 3, p. 178-191, 2013.

GARCIA, D. Efectos biológicos de la fragmentación de hábitats: nuevas aproximaciones para resolver un viejo problema. Ecosistemas, v. 20, n. 2, p. 1-10, 2011.

GARCIA, L. S.; SANTOS, A. M.; FOTOPOULOS, I. G.; FURTADO, R. S. Fragmentação florestal e sua influência sobre a fauna: estudo de caso na Província Ocidental da Amazônia, município de Urupá, estado de Rondônia. In: Simpósio Brasileiro de Sensoriamento Remoto, 16., Anais... SBSR, p. 3163-3170, 2013.

HENTZ, A. M. K. Mapeamento, fragmentação florestal e influência das áreas de borda para a comunidade arbórea no Alto Iguaçu - estado do Paraná. 2015. 197 f. Dissertação (Mestrado em Engenharia Florestal) - Universidade Federal do Paraná, Curitiba, 2015.

HENTZ, A.; CORTE, A. P. D; BetinA, D.; SANQUeTtA, C. R. Avaliação da fragmentação dos remanescentes florestais da bacia hidrográfica do Rio Iguaçu - PR, Brasil. Enciclopédia Biosfera, v. 11, n. 21, p. 2842-2858, 2015.

HENTZ, A.; CORTE, A. P. D; BLUM, C. T.; SANQUETTA, C. R. Técnicas orientada ao objeto para levantamento da fragmentação florestal na sub-bacia Alto Iguaçú, Paraná. Geosciências, v. 34, n. 4, p. 883896, 2015.

ITCG - Instituto de Terras, Cartografia e Geologia do Paraná. Clima - estado do Paraná. 2008. Disponível em: <http://www.itcg.pr.gov.br/arquivos/File/Produtos DGEO/Map as ITCG/PDF/Mapa Climas A3.pdf> Acesso em: 02/05/2017.

ITCG - Instituto de Terras, Cartografia e Geologia do Paraná. Formações fitogeográficas - estado do Paraná. 2009. Disponível em: <http://www.itcg.pr.gov.br/arquivos/File/Produtos DGEO/ Mapas ITCG/PDF/Mapa Fitogeografico A3.pdf> Acesso em: 02/05/2017.

INPE - Instituto Nacional de Pesquisas Espaciais. Catálogo de Imagens. 2017. Disponível em: 〈http://www.dgi.inpe.br/CDSR> Acesso em: 02/07/2016.

JUVANHOL, R. S; FIEDLER, N. C.; SANTOS, A. R.; PIROVANI, D. B.; LOUZADA, F. L. R. O.; DIAS, H. M.; TEBALDI, A. L. C. Análise espacial de fragmentos Florestais: caso dos parques estaduais de Forno Grande e Pedra Azul. estado do Espírito Santo. Floresta e Ambiente, v. 18, n. 4, p. 353-364, 2011.
MARUJO, R. F. B. Avaliação de métodos de segmentação de imagens aplicadas na classificação de culturas cafeeiras. 2013. 50 f. Monografia (Graduação em Ciência da Computação.) Universidade Federal de Lavras, Lavras, 2013.

MAPBIOMAS - Projeto de Mapeamento Anual da Cobertura e Uso do Solo no Brasil. Estatísticas. 2017. Disponível em: <http://mapbiomas.org/stats> Acesso em: 27/05/2017.

RAMBALDI, D. M.; OLIVEIRA, D. A. S. Fragmentação de ecossistemas: causas, efeitos sobre a biodiversidade $\mathrm{e}$ recomendações de políticas públicas. Brasília. Ministério do Meio Ambiente, 2003. 510 p.

SUDERHSA - Instituto das Águas do Paraná. Dados para download: unidades hidrográficas do Paraná. 2006. Disponível em: <http://www.aguasparana.pr.gov.br/modules/conteudo/ conteudo.php?conteudo=79> Acesso em: 27/05/2017.

SEMA - Secretaria Do Meio Ambiente e Recursos Hídricos. Bacias hidrográficas do Paraná: uma série histórica. 2013. Disponível em: <http://www.aguasparana.pr.gov.br/arquivos/File/BACIAS/ iguacu.pdf $>$ Acesso em: 12/05/2017.

PIROVANI, D. B.; SILVA, A. G.; SANTOS, A. R.; CECÍlIO, R. A.; GLERIANI, J. M.; MARTINS, S. V. Análise espacial de fragmentos florestais na Bacia do Rio Itapemirim, ES. Revista Árvore, v. 38, n. 2, p. 271-281, 2014.

RIBEIRO, M. C.; METZGER, J. P.; MARTENSEN, A. C.; PONZONI, F. J.; HIROTA, M. M. The Brazilian Atlantic forest: how much is left, and how is the remaining forest distributed? Implications for conservation. Biological Conservation, v. 142, p. 1141-1153, 2009.

RUDOLPHO, L. S.; BRAGHIROLLI, G.; REFOSCO, J. C.; SANTIAGO, A. G.; SABOYA, R. T. Aplicação de técnicas de geoprocessamento e métricas de paisagem na análise temporal da cobertura florestal da Bacia do Ribeirão Fortaleza em Blumenal/SC. In: Simpósio Brasileiro de Sensoriamento Remoto, 16., Anais... SBSR, p. 17421749, 2013.

SILVA, M. S. F.; SOUZA, R. M. Padrões espaciais de fragmentação florestal na Flona do Ibura - Sergipe. Mercator, v. 13, n. 3, p. 121-137, 2014.

UNFCCC - United Nation Framework Convention on Climate Change. Report of the conference of the parties on its seventh session, held at Marrakesh from $\mathbf{2 9}$ October to 10 November 2001. UNFCCC, 69 p. Disponível em: <http://unfccc.int/ resource/docs/cop7/13a01.pdf> Acesso em: 13/06/2017. 